\title{
Successive PM-based 2D DOA estimation for non-uniform L-shaped array
}

\author{
Lv Weihua ${ }^{1,2}$, Cheng Qianlin ${ }^{1}$, Zheng Wang $^{1}$, Zhang Xiaofei ${ }^{1,3, a}$ \\ ${ }^{1 .}$ College of Electronic and Information Engineering, Nanjing University of Aeronautics and \\ Astronautics, Nanjing, China, 210016 \\ 2. Luoyang Optoelectro technology development center, Luoyang, China, 471000 \\ 3. National Mobile Communications Research Laboratory, Southeast University, China, 210096 \\ aEmail: zhangxiaofei@nuaa.edu.cn
}

Keywords: DOA, Successive, Non-uniform L-shaped array, PM

\begin{abstract}
Propagator Method (PM) algorithm is a common method for the estimation of direction of arrival (DOA). As PM algorithm has no eigenvalue decomposition of the covariance matrix which is needed for MUSIC algorithm, it already has a lower computational complexity relatively. In this paper, we proposed a successive DOA estimation based on PM algorithm to lower the complexity further. Extensive simulations have been conducted to verify the usefulness of the proposed algorithm.
\end{abstract}

\section{Introduction}

Direction of arrival (DOA) estimation, a key technology in array signal processing, has been widely applied in communication, radar, sonar etc.. Recently, two-dimensional (2-D) direction of arrival (DOA) estimation for non-uniform L-shaped array has become a hot spot [1-2].

An improved PM method [3] for two parallel uniform linear arrays(ULA)[4] improves the performance in low SNR. Also, in Ref.[5] a propagator method (PM) based on L-shaped arrays has been proposed. However, the eigenvalue decompositions make the eigenvalues arranged arbitrarily. Ref.[6] addresses the joint singular value decomposition (SVD) method which can automatically pair for 2-D angle estimation, but they are computationally intensive because of using SVD. This paper proposes an successive algorithm based on PM algorithm for non-uniform L-shaped array which can significantly reduce the computational complexity with an acceptable performance.

Notations: Lower-case (upper-case) boldface symbols denote vectors (matrices). $(\bullet)^{T},(\bullet)^{H},(\bullet)^{-1}$, $(\bullet)^{+}$denote the transpose, the conjugate transpose, the inverse and the pseudo inverse, respectively. $E(\bullet)$ denotes the expectation operator.

\section{Data model}

In Fig.1, the non-uniform L-shaped array is composed of two linear subarrays with $M$ and $N$ omnidirectional sensors.

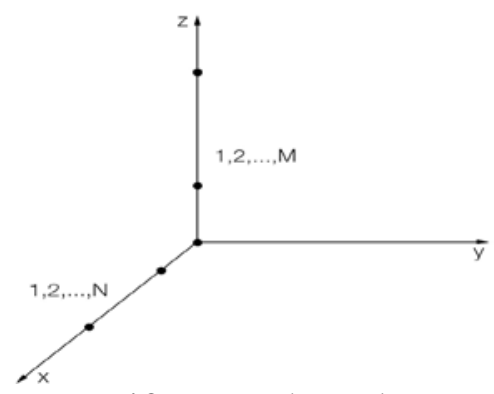

Fig.1 Non-uniform L-shaped array model 
Assuming there are $K$ uncorrelated narrowband far-field signals with the elevation angle $\theta_{k}$ and the azimuth angle $\phi_{k}$ of the $k$ th source $(k=1,2, \cdots, K)$ impinging on the non-uniform L-shaped array. The sensors are located at $\mathbf{d} x=\left[d_{x 1}, d_{x 2}, \cdots, d_{x N}\right]$ in $x$-axis and $\mathbf{d} z=\left[d_{z 1}, d_{z 2}, \cdots, d_{z M}\right]$ in z-axis.

The received signal of each subarray can be represented as

$$
\begin{aligned}
& \mathbf{Z}(t)=\mathbf{A}_{z} \mathbf{S}(t)+\mathbf{N}_{z}(t) . \\
& \mathbf{X}(t)=\mathbf{A}_{x} \mathbf{S}(t)+\mathbf{N}_{x}(t) .
\end{aligned}
$$

where $\mathbf{S}(t) \in C^{K \times L}, \quad \mathbf{N}_{x}(t) \in C^{N \times L}, \quad \mathbf{N}_{z}(t) \in C^{M \times L}, \mathbf{A}_{z}=\left[\mathbf{a}_{z}\left(\theta_{1}, \phi_{1}\right), \mathbf{a}_{z}\left(\theta_{2}, \phi_{2}\right), \cdots, \mathbf{a}_{z}\left(\theta_{K}, \phi_{K}\right)\right]$, $\mathbf{A}_{x}=\left[\mathbf{a}_{x}\left(\theta_{1}, \phi_{1}\right), \mathbf{a}_{x}\left(\theta_{2}, \phi_{2}\right), \cdots, \mathbf{a}_{x}\left(\theta_{K}, \phi_{K}\right)\right] \quad, \quad \mathbf{a}_{z}\left(\theta_{k}, \phi_{k}\right)=\left[e^{-j 2 \pi d_{z 1} \cos \theta_{k} / \lambda}, \cdots, e^{-j 2 \pi d_{z M} \cos \theta_{k} / \lambda}\right]^{T} \quad$ and $\mathbf{a}_{x}\left(\theta_{k}, \phi_{k}\right)=\left[e^{-j 2 \pi d_{x 1} \sin \theta_{k} \cos \phi_{k} / \lambda}, \cdots, e^{-j 2 \pi d_{x N} \sin \theta_{k} \cos \phi_{k} / \lambda}\right]^{T} . \mathbf{N}_{z}(t)$ and $\mathbf{N}_{x}(t)$ are additive white Gaussian noise vectors whose elements have mean zero and variance $\sigma^{2} . \lambda$ denotes the wavelength.

\section{The successive $P M$ algorithm}

\subsection{The first one-dimensional search}

The steering matrix $\mathbf{A}_{z}$ can be divided as

$$
\mathbf{A}_{z}=\left[\begin{array}{l}
\mathbf{A}_{1} \\
\mathbf{A}_{2}
\end{array}\right] \text {. }
$$

where $\mathbf{A}_{1} \in C^{K \times K}$ and $\mathbf{A}_{2} \in C^{(M-K) \times K}$.

The propagation operator $\mathbf{P}_{z} \in C^{K \times(M-K)}$ is defined as

$\mathbf{P}_{z}{ }^{H} \mathbf{A}_{1}=\mathbf{A}_{2}$.

$$
\left[\mathbf{P}_{z}{ }^{H}, \quad-\mathbf{I}_{M-K}\right] \mathbf{A}_{1}=\mathbf{Q}_{z}{ }^{H} \mathbf{A}_{1}=\mathbf{0} .
$$

Partition the extended covariance matrix $\mathbf{R}_{z}=\mathbf{Z}(t) \mathbf{Z}^{H}(t) / L$ as

$$
\mathbf{R}_{z}=\left[\begin{array}{ll}
\mathbf{G}_{z} & \mathbf{H}_{z}
\end{array}\right] \text {. }
$$

where $\mathbf{G}_{z} \in C^{M \times K}$ and $\mathbf{H}_{z} \in C^{M \times(M-K)}, L$ denotes the number of snapshots.

Propagation operator can be denoted as

$\hat{\mathbf{P}}_{z}=\left(\mathbf{G}_{z}{ }^{H} \mathbf{G}_{z}\right)^{-1} \mathbf{G}_{z}{ }^{H} \mathbf{H}_{z}$.

So we can obtain $\hat{\mathbf{Q}}_{z}{ }^{H}=\left[\hat{\mathbf{P}}_{z}{ }^{H},-\mathbf{I}_{2 M-K}\right]$.

Considering introducing $\hat{\mathbf{Q}}_{z}$ into the projection operator of the noise subspace, we replace it with its orthogonalized matrix $\hat{\mathbf{Q}}_{z 0}=\hat{\mathbf{Q}}_{z}\left(\hat{\mathbf{Q}}_{z}{ }^{H} \hat{\mathbf{Q}}_{z}\right)^{-1 / 2}$ and we can also get $\hat{\mathbf{Q}}_{z 0}^{H} \mathbf{a}_{z}(\theta)=\mathbf{0}$. Thus the pseudo spectrum function is given by

$$
f_{z}\left(\theta_{k}\right)=\mathbf{a}_{z}{ }^{H}\left(\theta_{k}\right) \mathbf{Q}_{z 0} \mathbf{Q}_{z 0}^{H} \mathbf{a}_{z}\left(\theta_{k}\right) .
$$

where $\mathbf{a}_{z}\left(\theta_{k}\right)=\left[e^{-j 2 \pi d_{z 1} \cos \theta_{k} / \lambda}, e^{-j 2 \pi d_{z 2} \cos \theta_{k} / \lambda}, \cdots, e^{-j 2 \pi d_{z M} \cos \theta_{k} / \lambda}\right]^{T}$.

By one-dimensional searching, the initial estimation of elevation angle $\hat{\boldsymbol{\theta}}_{i n i}=\left[\hat{\theta}_{i 1}, \hat{\theta}_{i 2}, \cdots, \hat{\theta}_{i K}\right]$ can be obtained.

\subsection{The second one-dimensional search}

In this section, we utilize the L-shaped array to implement the second one-dimensional search with the initial estimation of elevation angle in 3.1. Partition the steering matrix $\mathbf{A}_{x z}=\left[\mathbf{A}_{x} ; \mathbf{A}_{z}\right]$ as 


$$
\mathbf{A}_{x z}=\left[\begin{array}{l}
\mathbf{A}_{3} \\
\mathbf{A}_{4}
\end{array}\right] .
$$

where $\mathbf{A}_{3} \in C^{K \times K}$ and $\mathbf{A}_{4} \in C^{(M+N-K) \times K}$.

The propagation operator $\mathbf{P}_{x z} \in C^{K \times(M+N-K)}$ is defined as

$$
\left[\mathbf{P}_{x z}{ }^{H}, \quad-\mathbf{I}_{M+N-K}\right] \mathbf{A}_{3}=\mathbf{Q}_{x z}^{H} \mathbf{A}_{3}=\mathbf{0} .
$$

The extended covariance matrix $\mathbf{R}_{x z}$ can be divided as

$$
\mathbf{R}_{x z}=\left[\begin{array}{l}
\mathbf{X}(t) \\
\mathbf{Z}(t)
\end{array}\right]\left[\begin{array}{l}
\mathbf{X}(t) \\
\mathbf{Z}(t)
\end{array}\right]^{H} / L=\left[\mathbf{G}_{x z}, \mathbf{H}_{x z}\right] .
$$

where $\mathbf{G}_{x z} \in C^{(M+N) \times K}$ and $\mathbf{H}_{x z} \in C^{(M+N) \times(M+N-K)}$.

Propagation operator can be estimated by

$\hat{\mathbf{P}}_{x z}=\left(\mathbf{G}_{x z}{ }^{H} \mathbf{G}_{x z}\right)^{-1} \mathbf{G}_{x z}{ }^{H} \mathbf{H}_{x z}$.

Then we can get $\hat{\mathbf{Q}}_{x z}{ }^{H}=\left[\hat{\mathbf{P}}_{x z}{ }^{H}, \quad-\mathbf{I}_{M+N-K}\right]$.

We replace it with its orthogonalized matrix $\hat{\mathbf{Q}}_{x z 0}=\hat{\mathbf{Q}}_{x z}\left(\hat{\mathbf{Q}}_{x z}{ }^{H} \hat{\mathbf{Q}}_{x z}\right)^{-1 / 2}$. Then construct the pseudo spectrum function as

$$
f_{x z 1}\left(\hat{\theta}_{i n}, \phi_{k}\right)=\mathbf{a}_{x z}^{H}\left(\hat{\theta}_{i n}, \phi_{k}\right) \mathbf{Q}_{x z 0} \mathbf{Q}_{x z 0}^{H} \mathbf{a}_{x z}\left(\hat{\theta}_{i n}, \phi_{k}\right) .
$$

where $\mathbf{a}_{x z}\left(\hat{\theta}_{i n}, \phi_{k}\right)=\left[\begin{array}{l}\mathbf{a}_{x}\left(\hat{\theta}_{i n}, \phi_{k}\right) \\ \mathbf{a}_{z}\left(\hat{\theta}_{i n}, \phi_{k}\right)\end{array}\right]$ and $\hat{\theta}_{i n} \in \hat{\boldsymbol{\theta}}_{i n i}$.

By one-dimensional search via $\phi_{k}$, we can get the estimation of azimuth angle $\hat{\boldsymbol{\Phi}}=\left[\hat{\phi}_{1}, \hat{\phi}_{2}, \cdots, \hat{\phi}_{K}\right]$.

\subsection{The third one-dimensional search}

Through the estimation of azimuth angle in 3.2, we can achieve the third one-dimensional search. The spatial spectrum function should be rewritten as

$$
f_{x z 2}\left(\theta_{k}, \hat{\phi}_{n}\right)=\mathbf{a}_{x z}^{H}\left(\theta_{k}, \hat{\phi}_{n}\right) \mathbf{Q}_{x z 0} \mathbf{Q}_{x z 0}^{H} \mathbf{a}_{x z}\left(\theta_{k}, \hat{\phi}_{n}\right) \text {. }
$$

where $\mathbf{a}_{x z}\left(\theta_{k}, \hat{\phi}_{n}\right)=\left[\begin{array}{l}\mathbf{a}_{x}\left(\theta_{k}, \hat{\phi}_{n}\right) \\ \mathbf{a}_{z}\left(\theta_{k}, \hat{\phi}_{n}\right)\end{array}\right]$ and $\hat{\phi}_{n} \in \hat{\mathbf{\Phi}}$.

Then the second estimation of elevation angle $\hat{\boldsymbol{\theta}}_{\mathrm{sec}}=\left[\hat{\theta}_{\mathrm{s} 1}, \hat{\theta}_{\mathrm{s} 2}, \cdots, \hat{\theta}_{\mathrm{sK}}\right]$ can be obtained by one-dimensional search via $\theta$.

\subsection{Analysis of computational complexity}

The complexity of 2D PM is $K(M+N)(M+N-K)+2 K^{2}(M+N)+K^{3}+2(M+N)(M+N$ $-K)^{2}+(M+N-K)^{3}+(M+N)^{2} L+l_{1} l_{2}(M+N)(M+N-K)$ and the one of the proposed algorithm is $M^{2} L+2 M K^{2}+K M(M-K)+2 M(M-K)^{2}+(M-K)^{3}+(M+N)^{2} L+K(M+N)(M+N-K)$ $+2 K^{2}(M+N)+2 K^{3}+2(M+N)(M+N-K)^{2}+(M+N-K)^{3}+\left(l_{1}+l_{2}\right)(M+N)(M+N-K)+l_{1} M(M$ $-K)$, where $l_{1}$ is the search number of the elevation angle and $l_{2}$ is the search number of azimuth angle. The complexity of the proposed algorithm is greatly lower than that of the 2D PM which is illustrated in Fig.2. 


\section{Simulation results}

In this section, we illustrate the performance of the successive PM algorithm for non-uniform L-shaped array. Suppose $K=2$ sources impinging on the array located at $\left(\theta_{1}, \phi_{1}\right)=\left(20^{\circ}, 45^{\circ}\right)$, $\left(\theta_{2}, \phi_{2}\right)=\left(40^{\circ}, 35^{\circ}\right)$. The root mean square error (RMSE) of the estimations is defined as $R M S E$ $=\sqrt{\frac{1}{S K} \sum_{s=1}^{S} \sum_{k=1}^{K}\left(\alpha_{k}-\hat{\alpha}_{k, s}\right)^{2}}$ where $S$ denotes the times of Monte-Carlo simulations and $\hat{\alpha}_{k, s}$ is the estimation of the $k$ th angle $\alpha_{k}$ for the sth trial $(S=200)$.

In Fig.3, we compare the two estimations of elevation angle where $M=N=8, L=100$. It is indicated that the second estimations are more accurate than the first estimations.

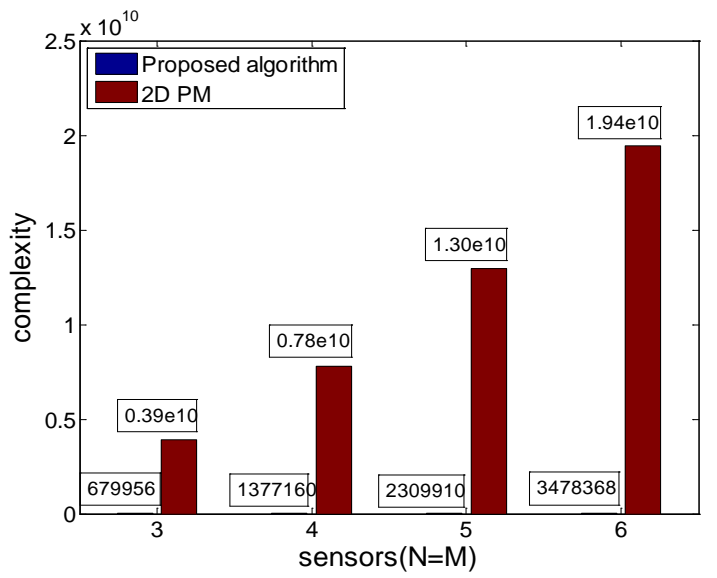

Fig.2 Comparison of computational complexity

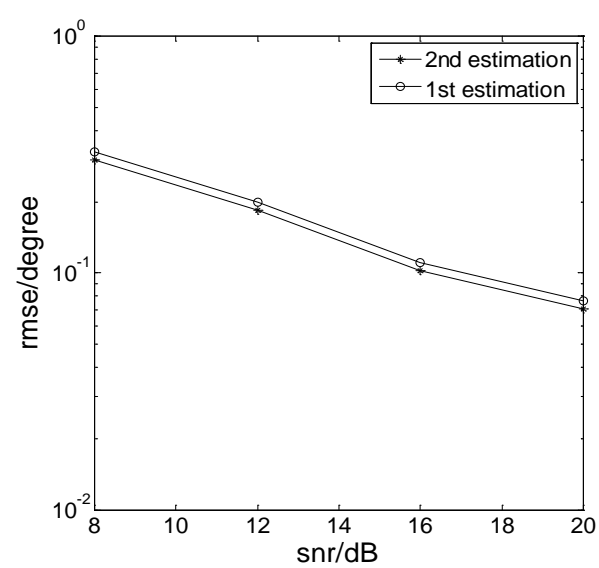

Fig.3 Comparison between the two estimations of elevation angles

Then, we change the number of sensors as shown in Fig. 4 where $L=100$. It is clearly indicated that the performance of proposed algorithm is getting better and better with the number of the sensors increasing because of diversity gain.

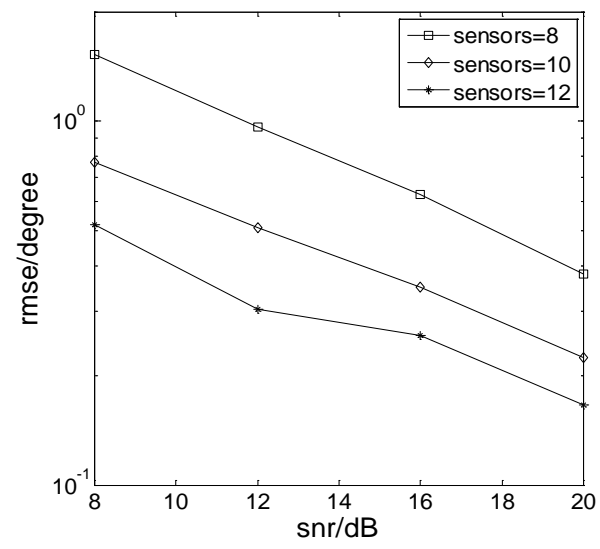

Fig.4 Performance of proposed algorithm with different number of sensors.

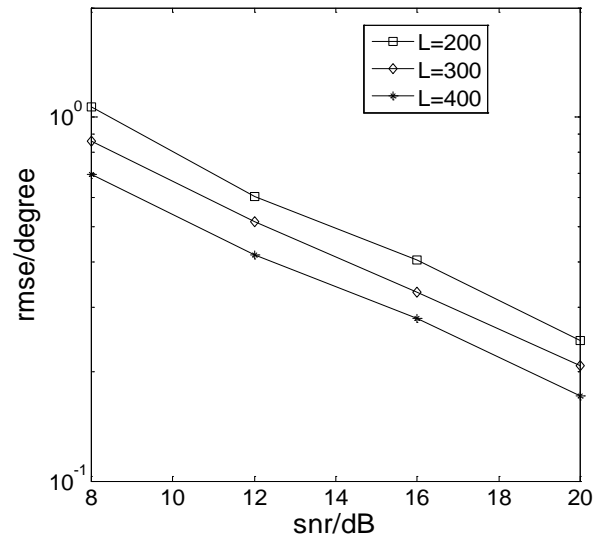

Fig.5 Performance of proposed algorithm with different snapshots.

We change the number of snapshots, where $M=N=8$ as shown in Fig.5. It is clearly indicated that the performance of the proposed algorithm is getting better and better with $L$ increasing because the larger the number of snapshots is, the more accurate the covariance matrix of the received data is. 


\section{Summary}

An effective method based on PM algorithm for non-uniform L-shaped array is proposed. It exploited three one-dimensional spectral peak searching to reduce the complexity. The simulation results showed that the successive PM algorithm for non-uniform L-shaped array can achieve satisfactory performances

\section{Acknowledgments}

This work is supported by China NSF Grants (61371169, 61271327), the open research fund of National Mobile Communications Research Laboratory, Southeast University (no. 2015D03), Funding for Outstanding Doctoral Dissertation in NUAA (BCXJ15-03), Qing Lan Project, and priority academic pro-gram development of Jiangsu high education institutions.

\section{References}

[1] J. Bach Andersen, Antenna arrays in mobile communications: gain, diversity, and channel capacity, IEEE Antennas and Propagation Magazine, Vol. 42, Issue 2, pp:12-16, 2000.

[2] E. Ben Dor, T. S. Rappaport, Qiao Yijun, et al, Millimeter-wave $60 \mathrm{GHz}$ outdoor and vehicle AOA propagation measurements using a broadband channel sounder, in Proceedings of the IEEE Global Telecommunications Conference, pp:1-6, 2011.

[3] Li Jianfeng, Zhang Xiaofei, Chen Han, Improved two-dimensional DOA estimation algorithm for two parallel uniform linear arrays using propagator method, Signal Processing, Vol. 92, Issue 12, pp:3032-3038, 2012.

[4] Bai L, Peng C Y, Biswas S. Association of DOA Estimation From Two ULAs [J]. IEEE Transactions on Instrumentation \& Measurement, vol.57, no.6, pp:1094-1101, 2008.

[5] N. Tayem, H. M. Kwon, L-shaped 2-dimensional arrival angle estimation with propagator method, IEEE Transactions on Antennas and Propagation, Vol.53, Issue 5, pp:1622-1630, 2005.

[6] Gu Jianfeng, Wei Ping, Joint SVD of two cross-correlation matrices to achieve automatic pairing in 2-D angle estimation problems, IEEE Antennas and Wireless Propagation Letters, Vol.6, pp: 553-556, 2007. 